\title{
EVALUATION AND ANALYSIS OF A MULTI-BAND TRANSCEIVER FOR NEXT GENERATION TELEMETRY APPLICATIONS
}

\author{
Nathan Richardson, D. Eng., Samuel Berhanu, \\ and Willie L. Thompson II, D.Eng \\ Morgan State University, The Clarence M. Mitchell Jr. School of Engineering \\ Center of Excellence for Tactical \& Advanced Communications Technologies
}

\begin{abstract}
This paper presents the evaluation and analysis of two multi-band, transceiver architectures that address the current demands in telemetry applications. One architecture used image rejection to perform coarse band selection, translating the RF spectrum to IF frequencies, while the second architecture utilizes an adaptive filter bank architecture for more agile band selectivity. Both architectures consist of an analog front-end subsystem for signal conditioning and frequency translation, and a digital radio subsystem for band and channel selection. The transceiver prototype was designed to operate over the L-band $(1435-1535 \mathrm{MHz})$, S-Band $(2200-2295$ $\mathrm{MHz}, 2310$-2385 MHz), and C-Band (4400 -4950 MHz, 5091 - $5250 \mathrm{MHz}$ ). System blockdiagrams, simulations and measured results with a comparison between the two architectures, are presented.

The authors would like to thank the Test Resource Management Center (TRMC) Test and Evaluation/Science and Technology (T\&E/S\&T) Program for their support. This works was funded by the T\&E/S\&T program through the U.S. Army Program Executive Office for Simulation, Training, and Instrumentation (PEO STRI) contract for W900KK-12-C-0048 for Multi-Band, Multi-Mode Software Defined Radio (MBMM SDR). This paper is approved for public release; distribution is unlimited (412TW-PA-14282).
\end{abstract}

\section{Introduction}

All sectors of the wireless community are challenged to provide its users fast, efficient means of transferring its continually growing volumes of data. The aeronautical telemetry infrastructure is no exception to this challenge. The growing pinch in the availability of spectrum has placed a demand for usage of the C-Band allocation for telemetry purposes. This in return has created a need for more band agile, multi-mode radios capable of fulfilling range telemetry applications. Moreover, the push for network-centric communication links requires radios with two-way communication capabilities.

\section{MBMM SDR System Description}

To address the current challenges and demands, the Center of Excellence for Tactical \& Advanced Communications Technologies (CETACT) at Morgan State University in Baltimore, Maryland has developed a multi-band, multi-mode software defined radio (MBMM SDR) transceiver [1]. This MBMM SDR consists of three main sub-systems: a multi-band front end 
supporting L/S/C-band telemetry allocations, a digital radio, and a configuration \& control subsystem. The multi-band front end (MBFE) provides tri-band operation, band selection, and channel tuning. The digital radio (DR) implements field-programmable gate array (FPGA) technology to provide high-speed signal processing and programmability to support multiple telemetry waveforms. The standard telemetry waveforms to be implemented are pulse code modulation/frequency modulation (PCM/FM) and shaped offset quadrature shift keying (SOQPSK-TG). The configuration and control (C2) sub-system allows for pre-test configuration and control of the multi-band front end and digital radio. This report provides an evaluation of the MBMM SDR to fulfill the requirements defined below.

\section{III.Multi-Band Front End Description, Evaluation \& Analysis}

The MBFE sub-system design consists of three sections: a wideband front end (WBFE) section, a RF adaptable filter bank (AFB) section, and a channel tuning (CT) section. Each section contains a transmitter $(\mathrm{Tx})$ and a receiver $(\mathrm{Rx})$ channel path, as illustrated in Figure 1 and Figure 2 for the respective implementations. Two (2) implementations of the CT section are presented: the Weaver CT implementation (CTW) and the adaptable filter bank CT implementation (CTA). The implementations differ in the methodology of translating the RF/IF signals. The general requirements guiding the design of each implementation is presented in Table 1.

Table 1: General Design Requirements

\begin{tabular}{|l|l|}
\hline Requirement Title & Requirement Statement \\
\hline Tri-Band Operation & $\begin{array}{l}\text { The MBFE shall perform with flat gain response across the } 500-6000 \mathrm{MHz} \\
\text { spectrum. } \\
\text { Minimum roll off or ripple in the gain response will be tolerated. }\end{array}$ \\
\hline Band Selection & $\begin{array}{l}\text { The MBFE shall possess the ability to select the appropriate spectrum for } \\
\text { transmission/reception enabling bi-directional communications. } \\
\text { The MBFE shall perform with high selectivity in order to reject adjacent } \\
\text { bands and to make the SDR electromagnetic interference (EMI) tolerant. }\end{array}$ \\
\hline Channel Tuning & $\begin{array}{l}\text { The translation section shall possess high port-to-port isolation to minimize } \\
\text { unintended transference of interfering signals. } \\
\text { The local oscillator (LO) shall possess the appropriate tuning capability to } \\
\text { ensure frequency translation of signals within the spectrum of interest. } \\
\text { The IF filter response shall be designed such that adjacent channels and } \\
\text { interfering signals are attenuated. }\end{array}$ \\
\hline
\end{tabular}

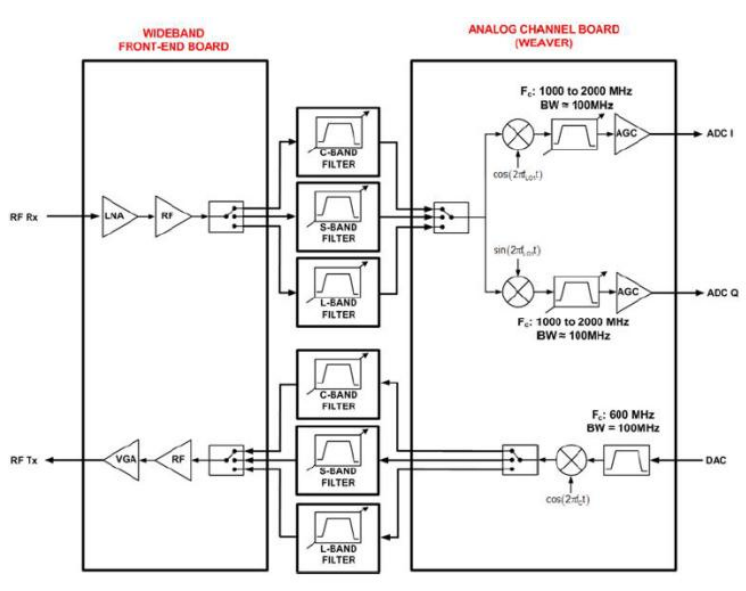

Figure 1: MFBE sub-system incorporating the Weaver implementation

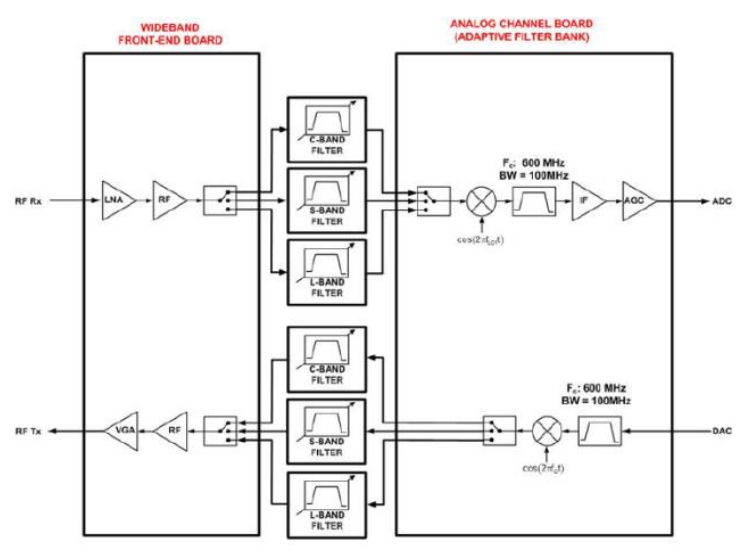

Figure 2: MBFE sub-system incorporating the AFB implementation 
Note, the evaluation presented herein show the capability of the prototypes to meet these general requirements. Evaluation of tri-band operational capability is presented via analysis of the insertion loss (IL) and return loss (RL) characteristics of the stage amplification. Band selection evaluation is presented via calculations of the selectivity performance of the appropriate filter components. Channel tuning evaluation is presented via calculation of the mixer's translation parameters - conversion loss, and port-to-port isolation, as well as, selectivity capabilities of the analog IF filters.

\section{Tri-Band Operation Evaluation: Wideband Front-End (WBFE)}

The WBFE section conditions the incoming RF signals for subsequent frequency in the Rx channel path, while conditioning the outgoing RF signal for final power amplification. Evaluation of the WBFE is to ensure that the appropriate signal conditioning (amplification) is achieved within the L/S/C-bands. Figure 3 and Figure 4 present a representation of the gain and return loss (RL) responses for the main amplification sections of the WBFE PCB. The measured responses presented are representative of the gain and RL performance of both the low noise amplifier (LNA) in the Rx channel and general gain block amplifiers (GBA) in both the Tx \& Rx channels. Figure 4 presents the measured gain response versus control voltage of the variable gain amplifier (VGA). As seen, the gain response in the LNA/GBA components is fairly flat with less than $2 \mathrm{~dB}$ of roll-off. Both the input and output return loss responses show good matching capability across the spectrum of interest. The VGA capabilities show $25 \mathrm{~dB}$ of gain variation, however, significant roll-off in the gain response across frequency is observed. This will have a dramatic effect on providing the final power amplifier consistent power across the L/S/C-Bands.

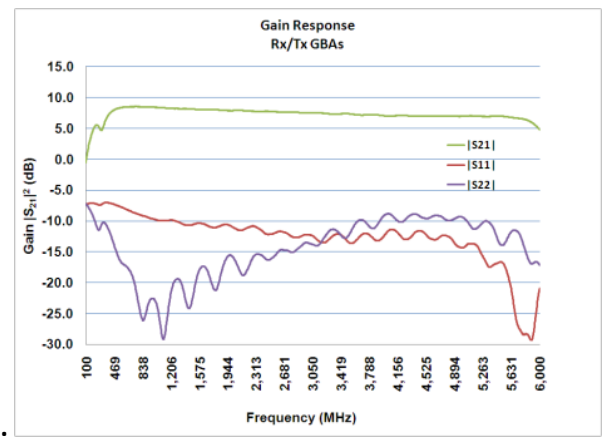

Figure 3: Representative gain and return loss responses for the LNA and GBA in the Rx and Tx channel paths

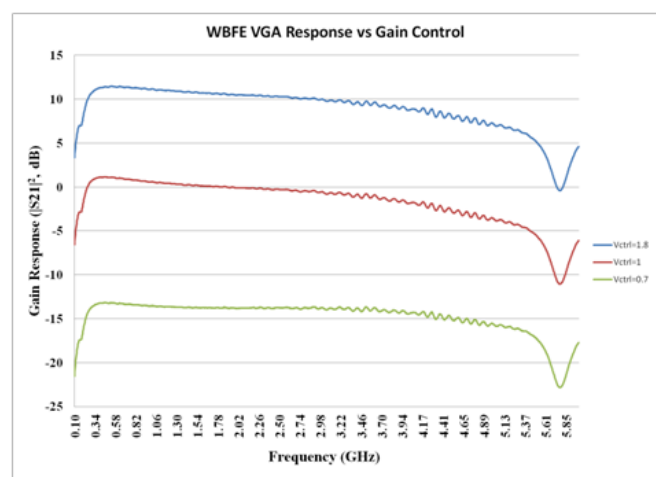

Figure 4: WBFE Tx VGA gain response versus control bias

\section{Analog Band Selection \& CT Evaluation \& Analysis: RF/IF Bandpass Filtering}

The capability of the MBMM SDR to fulfill the analog band selection and coarse channel tuning requirements is achieved in the RF AFB section, as well as, the IF filter stage of the CT implementations. The RF AFB section contains three (3) adaptable filters (L/S/C Bands) for both the $\mathrm{Rx} / \mathrm{Tx}$ channel paths. These commercially available filters enable tuning of the appropriate band filter response via the supply of control voltage signal for center frequency $\left(f_{c}\right)$ and a bandwidth (BW) adjustment. These filters are tuned to capture $100 \mathrm{MHz}$ of spectrum within the pre-selected band. In the CT section, after the frequency translation mixer, the IF filter stage contains an adaptable (CTW) band pass filter (ABPF) or fixed (CTA) band pass filter (BPF). The CTW ABPF is similar in functionality to the RF ABPFs, except here the $100 \mathrm{MHz}$ spectrum is contained within the 1000 to $2000 \mathrm{MHz}$ IF band. The CTA fixed IF BPF is designed with a $\mathrm{f}_{\mathrm{C}}$ of 
$600 \mathrm{MHz}$, a passband $\mathrm{BW}$ of $100 \mathrm{MHz}$, and rejection of $50 \mathrm{dBc}$ at frequencies less than 500 $\mathrm{MHz}$ and greater than $700 \mathrm{MHz}$.

Figure 5 and Figure 6 provide the filter responses for these respective filter components. Note, the ability of the ABPF components to capture the $100 \mathrm{MHz}$ spectrum is limited by the fractional bandwidth control. This has a profound impact on the band selection and coarse channel tuning capabilities to be demonstrated below. In the CTA implementation, the fixed IF BPF response shows excellent passband and rejection $(\sim 45 \mathrm{dBc})$ capabilities.

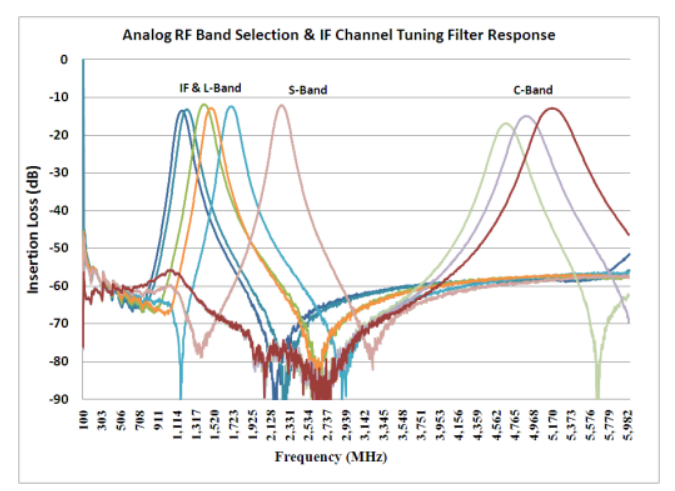

Figure 5: Analog RF Band and IF Channel Selection filter responses.

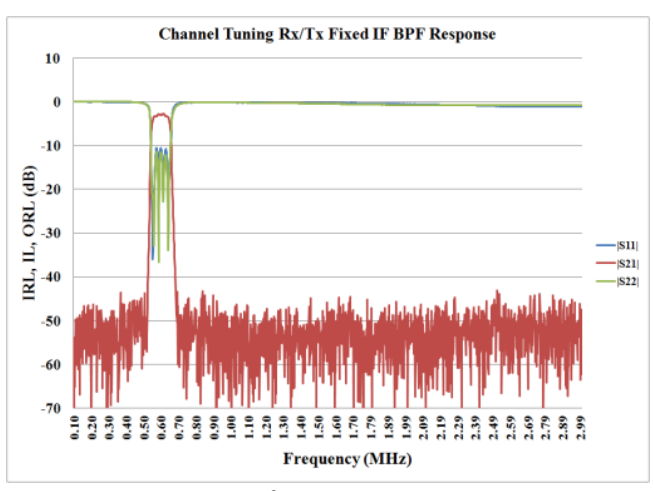

Figure 6: Rx IF BPF/Amp cascaded responses

\section{Channel Tuning Evaluation \& Analysis: CTA Rx/Tx Implementation}

The CTA implementation uses a frequency translation stage within the analog channel after the adaptive filter bank section. In the Tx channel path a $600-\mathrm{MHz}$ IF signal is translated up to the appropriate telemetry band (Tx channel path); while, in the Rx channel path, the selected telemetry band signal is translated band down to the $600 \mathrm{MHz}$ IF band. The frequency translation strategy is guided by the frequency plan presented in Figure 8. The evaluation of the CTA channel tuning section will now be presented. The evaluation will provide conversion loss and port-to-port isolation performances under specific translation modes, as well as, will augment the band-selection performance.

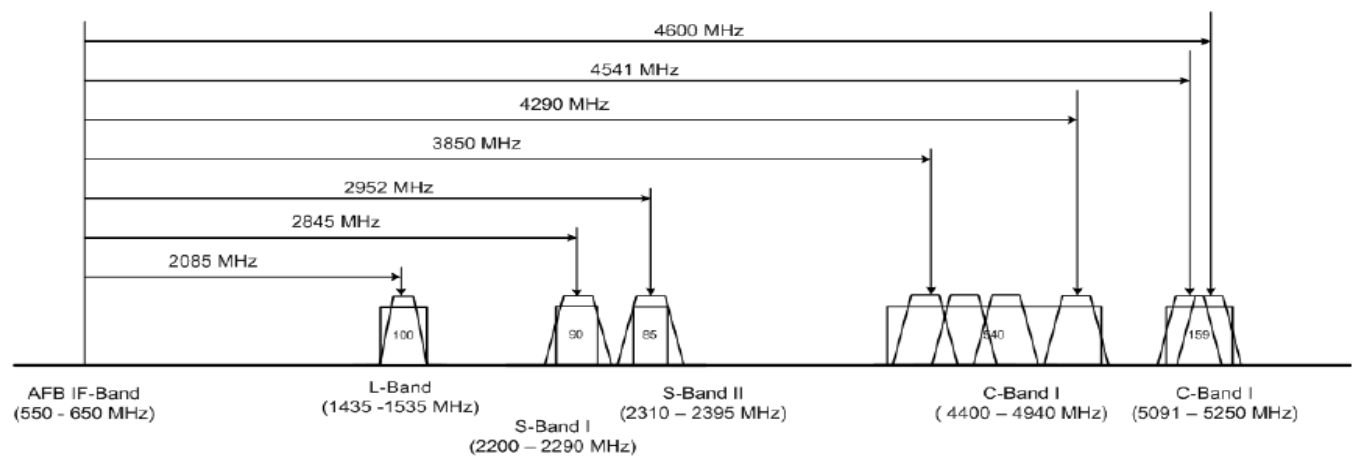

Figure 7: Frequency plan guiding the CTA Rx/Tx translation design.

To evaluate mixer translation and selectivity capabilities, the output spectrum of the mixer stage is captured using a spectrum analyzer. For each $\mathrm{Rx} / \mathrm{Tx}$ mode, the input specifications for the $\mathrm{LO} / \mathrm{RF}$ and LO/IF are given in Table 2. The input specifications and the output spectrum are used to calculate the mixer metrics according to the following [2]: 
Table 2: Mixer stage input specifications.

\begin{tabular}{|c|c|}
\hline Stage Input & Power Level \\
\hline$\left[\mathrm{P}_{\mathrm{RF} \_ \text {in }}\right]_{\mathrm{dB}} /\left[\mathrm{P}_{\mathrm{IF} \_ \text {in }}\right]_{\mathrm{dB}}$ & $-10 \mathrm{dBm}$ \\
\hline$\left[\mathrm{P}_{\mathrm{LO} \_\mathrm{in}}\right]_{\mathrm{dB}}$ & $+15 \mathrm{dBm}$ \\
\hline
\end{tabular}

$$
\begin{array}{ll}
C L_{d B}=P_{R F_{-} d B}-P_{I F_{-} d B} & \text { Eq. } 1 \\
{\left[P_{R F}\right]_{d B}=\left[P_{R F_{-} i n}\right]_{d B}-\left[L_{\text {int } \_R F}\right]_{d B}} & \text { Eq. } 2 \\
{\left[P_{L O}\right]_{d B}=\left[P_{L O_{-} i n}\right]_{d B}-\left[L_{\text {int } \_} L\right]_{d B}} & \text { Eq. } 3 \\
{\left[P_{I F}\right]_{d B}=\left[P_{I F_{-} i n}\right]_{d B}+\left[L_{\text {int_IF }}\right]_{d B}} & \text { Eq. } 4
\end{array}
$$

$\left[\mathrm{P}_{\mathrm{XY} \_}\right]_{\mathrm{dB}}$ is the power supplied to the $\mathrm{XY}$ port of the mixer stage, $\left[\mathrm{P}_{\mathrm{XY}}\right]_{\mathrm{dB}}$ is the power at the mixer's reference, and $\left[\mathrm{L}_{\mathrm{int} \_X Y}\right]_{\mathrm{dB}}$ are the internal losses between the stage inputs and the device's reference.

$$
\begin{aligned}
& {\left[I S O l_{R F_{-} I F}\right]_{d B}=\left[P_{R F}\right]_{d B}-\left[P_{I F_{-} R F}\right]_{d B}} \\
& {\left[I S o l_{L O_{-} I F}\right]_{d B}=\left[P_{L O}\right]_{d B}-\left[P_{I F_{-} L O}\right]_{d B}}
\end{aligned}
$$

$\left[\mathrm{P}_{\mathrm{IF} \_\mathrm{XY}}\right]_{\mathrm{dB}}$ is the power observed at the IF reference oscillating at the respective $\mathrm{XY}$ frequency.

Table 3 and Table 4 below summarize the channel tuning capability of the of the CTA Rx/Tx. The CTA Rx shows consistent conversion loss, port-to-port isolation and selectivity performance across each band. The optimum selectivity is limited by the LO-IF isolation performance. The average $45 \mathrm{dBc}$ selectivity meets the goal $(>40 \mathrm{dBc})$.

Table 3: Summary of the CTA Rx translation and spectrum selectivity capability.

\begin{tabular}{|c|c|c|c|c|}
\hline Rx Mode & $\begin{array}{c}\text { Conversion Loss } \\
(\mathbf{C L})\end{array}$ & $\begin{array}{c}\text { RF-IF } \\
\text { Isolation }\end{array}$ & $\begin{array}{c}\text { LO-IF } \\
\text { Isolation }\end{array}$ & Selectivity \\
\hline CTA Rx L-Band & $8.6 \mathrm{~dB}$ & $37 \mathrm{~dB}$ & $20 \mathrm{~dB}$ & $45 \mathrm{dBc}$ \\
\hline CTA Rx S-Band & $8.6 \mathrm{~dB}$ & $40 \mathrm{~dB}$ & $21 \mathrm{~dB}$ & $\sim 44 \mathrm{dBc}$ \\
\hline CTA Rx C-Band & $8.7 \mathrm{~dB}$ & $38 \mathrm{~dB}$ & $21.7 \mathrm{~dB}$ & $\sim 45 \mathrm{dBc}$ \\
\hline
\end{tabular}


While the conversion loss, and LO-RF isolation performance is consistent across the band allocations, the band selection capability of the CTA Tx does not meet the goal $(>40 \mathrm{dBc})$, especially at $\mathrm{C}$-Band. This degradation is a result of the fractional bandwidth capability of the ABPFs. The fractional bandwidth control of these commercial components is limited to the range of $11 \%$ for wide-bandwidth capture and 3\% for narrow bandwidth capture. For the C-Band ABPFs this only enables capturing as low as $120 \mathrm{MHz}$ of spectrum. At the narrow bandwidth range, the insertion loss increases dramatically, placing a requirement for adaptive amplification across the RF/IF bands.

Table 4: Summary of the CTA Tx translation and spectrum selectivity capability.

\begin{tabular}{|c|c|c|c|c|}
\hline Rx Mode & $\begin{array}{c}\text { Conversion Loss } \\
(\text { CL) }\end{array}$ & $\begin{array}{c}\text { IF-RF } \\
\text { Isolation }\end{array}$ & $\begin{array}{c}\text { LO-RF } \\
\text { Isolation }\end{array}$ & Selectivity \\
\hline CTA Tx L-Band & 8.6 & $30 \mathrm{~dB}$ & $21 \mathrm{~dB}$ & $\sim 30 \mathrm{dBc}$ \\
\hline CTA Tx S-Band & $8.7 \mathrm{~dB}$ & $35 \mathrm{~dB}$ & $23 \mathrm{~dB}$ & $33 \mathrm{dBc}$ \\
\hline CTA Tx C-Band & $8.7 \mathrm{~dB}$ & $25 \mathrm{~dB}$ & $26 \mathrm{~dB}$ & $22 \mathrm{dBc}$ \\
\hline
\end{tabular}

\section{Channel Tuning Evaluation \& Analysis: Weaver Implementation Rx Channel}

The CTW channel tuning implementation leverages the image-rejection property to perform band selection between the telemetry allocations [1]. The two-stage frequency translation architecture generates in-phase (I) and quadrature (Q) spectrum components with phase differences relative to each other. These phase differences allows for the selection between the upper sideband (USB) and the lower sideband (LSB) components. The first frequency translation is performed in the analog domain. This analog stage translates the RF energy to an intermediate frequency equi-distant between the two bands in the respective mode. The evaluation of this subsystem PCB will provide the capability to down-convert the RF energy, to properly capture the IF energy while rejecting potential interferers created by the translation process, and to condition the captured signal for later analog-to-digital (ADC) conversion.

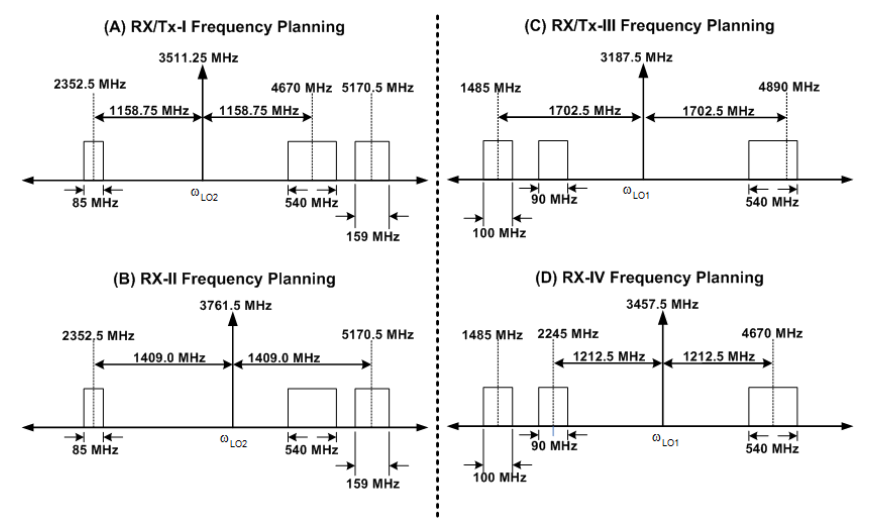

Figure 8: Frequency plan for the Weaver CT implementation

Table 5 summarizes the translations and channel selection capabilities of the CTW implementation. Similar to the CTA Tx, the selection capability is again limited by the selection capability of the IF ABPF components. Further investigation of the optimal tuning of these filters is required.

Table 5: Summary of the mixer metrics and filter selectivity for each mode of the Weaver implementation.

\begin{tabular}{|c|c|c|c|c|}
\hline Rx/Tx Mode & $\begin{array}{c}\text { Conversion Loss } \\
\text { (CL) }\end{array}$ & $\begin{array}{c}\text { RF-IF } \\
\text { Isolation }\end{array}$ & $\begin{array}{c}\text { LO-IF } \\
\text { Isolation }\end{array}$ & Selectivity \\
\hline
\end{tabular}




\begin{tabular}{|c|c|c|c|c|}
\hline I & $8.6 \mathrm{~dB}$ & $37 \mathrm{~dB}$ & $20 \mathrm{~dB}$ & $28 \mathrm{dBc}$ \\
\hline II & $8.6 \mathrm{~dB}$ & $40 \mathrm{~dB}$ & $21 \mathrm{bB}$ & $\sim 44 \mathrm{dBc}$ \\
\hline III & $8.7 \mathrm{~dB}$ & $38 \mathrm{~dB}$ & $21.7 \mathrm{bB}$ & $\sim 37 \mathrm{dBc}$ \\
\hline IV & 8.6 & $42 \mathrm{~dB}$ & $21 \mathrm{~dB}$ & $\sim 30 \mathrm{dBc}$ \\
\hline
\end{tabular}

\section{Digital Radio Description, Evaluation \& Analysis}

The digital radio firmware supports the transmitter and the receiver chain for both the Weaver and Adaptive Filter Bank architectures. The transmitter design for the Weaver architecture remains the same as that of the Adaptive Filter Bank architecture. The differences between the architectures are pronounced in the receiver path chains.

Figure 9 and Figure 9: Weaver Architecture

Figure 10 show the system block diagrams for the Weaver and Adaptive Filter Bank (AFB) digital firmware architectures.

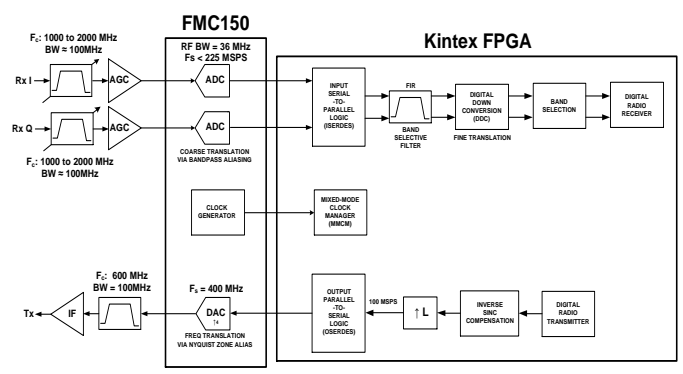

Figure 9: Weaver Architecture

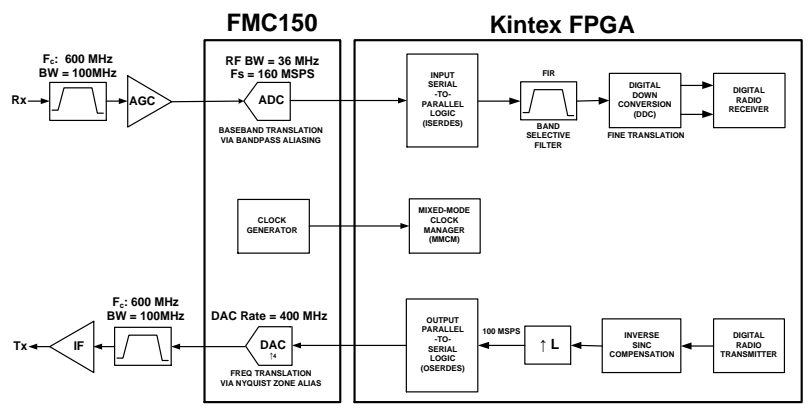

Figure 10: AFB Architecture

As shown in both figures, the digital firmware interface to the RF analog circuitry is accomplished through an FMC 150 card. The AFB Rx chain uses only one ADC as the signal received from the corresponding analog circuitry is real; the Weaver Rx chain uses both ADCs.

Tx Chain: For the transmitter chain, both the Weaver and Adaptive Filter Bank architectures incorporate, an inverse-sync compensation, interpolation stages for rate matching, and final conversion from digital to analog. Baseband-to-IF translation is accomplished via a Nyquist zone replica that is created during the conversion process. A frequency translation scheme was devised where the 2nd Nyquist zone replicas from the digital-to-analog converter (DAC) output was chosen to serve as the IF input to the analog channel board. To ensure that the digital signal would translate to $600 \mathrm{MHz}$, the $\mathrm{DAC}$ rate and $\mathrm{ADC}$ rate were configured to be 400 MSPS and 225 MSPS, respectively. The frequency translation of the baseband signal to 600MHz IF is demonstrated in Figure 11. 


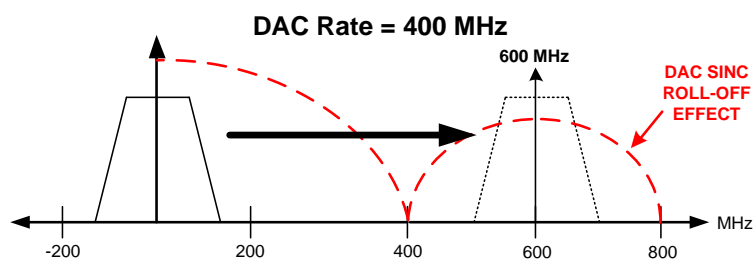

Figure 11: Tx Chain Digital Frequency Translation

Several Tx digital chain interpolation configurations are implemented to support 100 MSPS at the final stage of the signal path before entering the DAC. The supported data rates include $1 \mathrm{Mbps}$, 5Mbps, $10 \mathrm{Mbps}$, $25 \mathrm{Mbps}$, and $50 \mathrm{Mbps}$. Each configuration mode consist interpolation filter stages to clock the samples arriving at the input of the DAC at 100-MSPS. The interpolation filter types consist of half-band, \& FIR interpolators. Note, a 4x interpolation stage is implemented within the DAC for each Tx chain configuration mode.

Rx Chain: The IF frequencies proposed for both the Weaver \& AFB architectures in Figure are converted with the sampling frequencies presented in XXX below. Since direct Nyquist sampling is not practical at the proposed IF frequencies, band pass sampling is used for conversion. Based on the 100-MHz IF bandwidth specification, the minimum sampling clock is $400 \mathrm{MHz}$ to ensure a minimum availability of 4 samples in every symbol received. In addition, the sampling rate mapped the spectrum to the center of the Nyquist zone. This corresponds to a phasor of 4 Samples/Cycle in the digital spectrum.

Table 6: Frequency Translation \& Data Conversion to Digital Domain for Weaver \& AFB Architectures

\begin{tabular}{|l|c|c|}
\hline Mode & IF(MHz) & Sampling Clock (MSPS) \\
\hline Weaver I & 1158.75 & 159.83 \\
\hline Weaver II & 1409 & 225.44 \\
\hline Weaver III & 1702.5 & 144.89 \\
\hline Weaver IV & 1215.5 & 194 \\
\hline AFB & 600 & 160 \\
\hline
\end{tabular}

Because band-pass sampling can map unwanted signals outside of the IF band of interest to within the Nyquist zone, a band selective filter is implemented to filter out any unwanted spectra that would exist outside of the pass band of the band selective filer. This FIR filter is available for both the Weaver and AFB Rx digital chains. The filter spans a $100 \mathrm{MHz} 3-\mathrm{dB}$ bandwidth, and a minimum of $40 \mathrm{~dB}$ attenuation in the stop band.

The location of the spectrum of interest from IF at the center of the digital Nyquist zone allows for a down conversion of the spectrum to baseband without an actual heterodyne. The magnitude for the complex heterodyne -0.25 cycles/sample stated above are limited in discrete values of $+1,0$, or -1 . This simplifies the operation of the coarse translation as actual complex mixing can be bypassed for a more simplified down conversion. The Weaver architecture coarse translation stage is designed within the methodology presented in [1]. The AFB architecture would also follow on from [1] with the requirement that only one quadrature signal processing pair is necessary for the incoming real analog signal.

Results: Weaver Architecture Band Selection 
For the first phase of the design outlined above, the weaver architecture has been examined for its performance in being able to select the transmission \& channel select. Two frequencies of transmission were used at $1485 \mathrm{MHz}$ (designated from here on forth as L-Band signal), and 4489 $\mathrm{MHz}$ (designated from here on forth as C-Band signal) at the transmission. The designation is corresponding to the upper and lower sided-band spectra existing in the methodology outlined in [1]. Two types of waveforms were used for testing purposes: a continuous wave with peak power of $5 \mathrm{dBm}$ and, a CPM signal at $4 \mathrm{MSymbol} / \mathrm{sec}$. Both signals were produced from an arbitrary waveform generator, which went through a connectorized RF circuitry to form the first stage RF down conversion.

As an example, Figure 12 shows the spectrum of a tone signal transmitted at $1485 \mathrm{MHz}$ at an IF frequency of $1702 \mathrm{MHz}$ after being sampled residing at the center of the Nyquist zone.

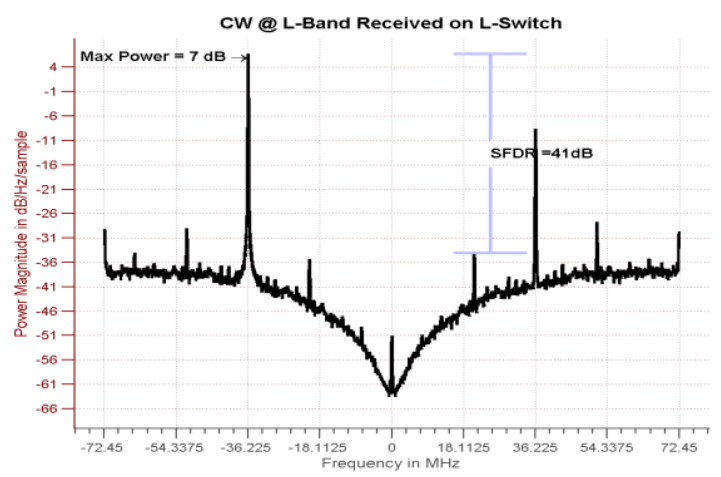

Figure 12: Spectrum of $\mathrm{CW}$ tone after sampling

Digital Image Rejection Performance: To determine the image rejection performance, the spectrum between the upper side band (L/S- band switch) and the lower side band spectrum (Cband Switch) from the digital Weaver channel Rx chain output were compared.

The first evaluation was based on the transmission of an L-Band tone and, reception of the lower side band (LSB) component (from toggling the L-Switch) in comparison to the upper side band (USB) component (C-Switch). The resulting spectra from the test are shown on Figure 13 below.

As shown on Figure 13, the suppression of the image signal, in this case the L-Switch spectrum, was close to $15 \mathrm{~dB}(36-19=15 \mathrm{~dB})$.

A similar test conducted with a C-Band tone transmission and the results showed that suppression on the order of $10 \mathrm{~dB}$ was achieved. Further tests conducted with modulated waveforms with the Weaver image rejection architecture consistently achieved a $10 \mathrm{~dB}$ suppression. 


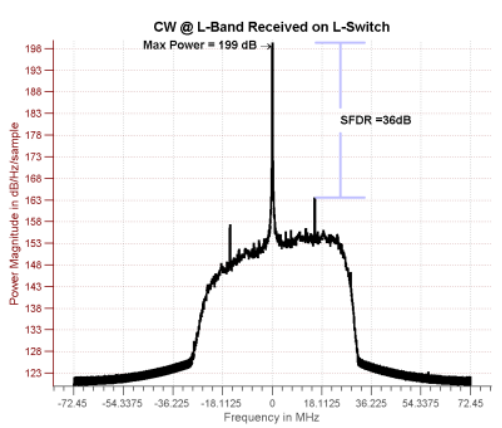

(a)

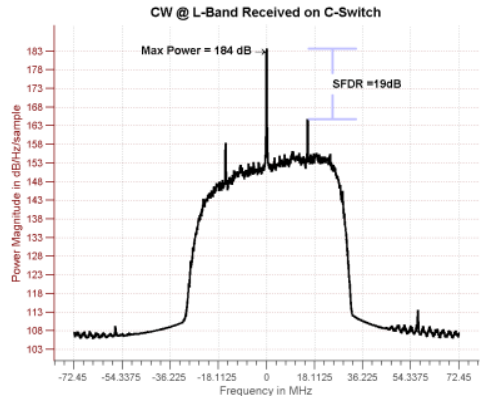

(b)

Figure 13: PSD Plots at the Weaver Receiver for L-Band Transmission (a) LSB side \& (b) USB,

All tests conducted above had I-Q imbalance, as the plots from Figure 13 indicate. This imbalance degraded the image rejection performance of the Weaver Rx architecture since perfect quadrature relationships couldn't be established between the I \& Q paths. Still, a suppression of $10 \mathrm{~dB}$ was achieved. Another factor that degraded the performance of the Rx Weaver channel was the FMC150. Though the daughter card was able to sample the signal at the IF frequencies, the full bandwidth specification for the card was limited to $500 \mathrm{MHz}$. This meant that at input frequencies of $1 \mathrm{GHz}$ and above, the sampler was not able to optimally capture the analog signal.

\section{Conclusion}

As summarized below, the MBFE met functional requirements for TRL4 Validation by providing wideband operation between 1 to $6 \mathrm{GHz}$, band selection between the C-,L- or Stelemetry bands, and the tuning of specific channels within the bands just mentioned.

In addition to validating the three specific areas, efforts were conducted to distinguish the two implementations (Weaver and AFB). Below are the summary findings of the Weaver vs. AFB comparison:

- Image rejection performance at sub optimum operation for the Weaver architecture was at least $10 \mathrm{~dB}$ and above.

- I\&Q balance mitigation circuitry is required for the Weaver implementation for correct demodulation.

- $\mathrm{AFB}$ is impacted by selectivity due to the fractional bandwidth performance of ABPF at higher frequencies.

\section{REFERENCES}

[1] Thompson II, Willie L. "A Multi-Band Transceiver Design For L/S/C-Band Telemetry," Proc. ITC/USA, pp. 538-546, San Diego, October 2012.

[2] Marki, Ferenc and Christopher Marki, " Mixer Basics Primer A Tutorial for RF \& Microwave Mixers", Marki Microwave, 2010. 